\title{
Ventilação mecânica volume-controlada versus pressão controlada em modelo canino de lesão pulmonar aguda: efeitos cardiorrespiratórios e sobre o custo de oxigênio da respiração*
}

Bruno do Valle Pinheiro ${ }^{1}$, Marcelo Alcântara Holanda ${ }^{2}$, Célia Mallart larges ${ }^{3}$, Oswaldo Shigueomi Beppu 4

Introdução: Persiste a questão sobre se há vantagens mecânicas ou de trocas gasosas no uso da ventilação pressão-controlada (VPC) sobre a ciclada a volume (VCV). Objetivos: Comparar, de forma randômica, a VPC com a VCV com fluxo desacelerado nos modos assistido e controlado em modelo experimental de lesão pulmonar aguda. Métodos: Sete cães com lesão pulmonar aguda grave $\left(\mathrm{PaO}_{2} /\right.$ $\left.\mathrm{FIO}_{2}<100 \mathrm{mmHg}\right)$ induzida por ácido oléico intravenoso $(0,05 \mathrm{mg} / \mathrm{kg})$ foram ventilados em VPC ou VCV, mantidos constantes o volume corrente e o tempo inspiratório. Nas duas modalidades os animais foram ventilados por 40 minutos no modo assistido seguido do modo controlado após curarização.

Resultados: Não houve diferenças em relação às trocas gasosas $\left(\mathrm{PaO}_{2}\right.$ e $\left.\mathrm{PaCO}_{2}\right)$, ao débito, ao transporte de oxigênio e à mecânica respiratória entre a VCV e a VPC. $\mathrm{O}$ consumo de oxigênio $\left(\mathrm{VO}_{2}\right)$ após a curarização foi semelhante (124 \pm 48 na vcV versus $143 \pm 50 \mathrm{ml} / \mathrm{min}$ na VPC, com $p=0,42$ ). Entretanto, no modo assistido, houve tendência de maior Vo ${ }_{2}$ na VPC $(219 \pm 72$ versus $154 \pm 67 \mathrm{ml} /$ min na $v C V, p=0,06$ ). Isso associou-se a tendência de maior custo de oxigênio da respiração (COR) naquela modalidade, embora sem diferença estatística significante (31 \pm 77 na vcv versus $75 \pm$ $96 \mathrm{ml} / \mathrm{min}$ na VPC, $p=0,23$ ) e menor $\mathrm{PvO}_{2}(34 \pm 7$ versus $42 \pm 6 \mathrm{ml} / \mathrm{min}$ na $v \mathrm{cv}, \mathrm{p}=0,02)$. 0 pico de fluxo inspiratório nos ciclos assistidos foi maior na VPC (58 \pm 9 versus $48 \pm 4 \mathrm{~L} / \mathrm{min}$ na VCV, $p=$

0,01). A instituição da ventilação controlada por curarização reduziu em cerca de $20 \%$ débito cardíaco e $\mathrm{O} \mathrm{DO}_{2}$ em relação ao modo assistido, tanto na VCV quanto na VPC. Conclusões: Em um modelo de insuficiência respiratória grave, com elevado COR, a manutenção da ventilação controlada

em relação à assistida melhorou a relação entre oferta e consumo de oxigênio. A VPC não trouxe benefícios às trocas gasosas ou à mecânica pulmonar em relação à VCV, podendo aumentar o COR no modo assistido no presente modelo. (J Pneumol 2002;28(1):15-22)

\section{Volume controlled ventilation versus pressure controlled ventilation in a canine acute lung injury model: effects on cardiorespiratory parameters and oxygen cost of breathing}

Background: It is questionable whether pressure-controlled ventilation (PCV) has advantages over volume-cycled ventilation ( $V C V$ ). Objectives: To compare PCV to VCV with decelerating flow profile during assisted and controlled modes in an acute lung injury experimental model. Methods: Severe acute lung injury $\left(\mathrm{PaO}_{2} / \mathrm{FIO}_{2}<100 \mathrm{mmHg}\right)$ was induced by oleic acid IV infusion $(0.05 \mathrm{mg} / \mathrm{kg})$ in seven dogs. The animals were submitted to PCV and VCV in a randomized sequence. After 40 minutes in the assisted mode, ventilation was changed to the controlled mode after neuromuscular blockade. The tidal volume and the inspiratory time were kept constant throughout the experiment. Results: There were no differences in gas exchange $\left(\mathrm{PaO}_{2}\right.$ and $\left.\mathrm{PaCO}_{2}\right)$, cardiac output or oxygen delivery $\left(\mathrm{DO}_{2}\right)$ between $V C V$ and PCV. The same was observed regarding

* Trabalho realizado na Disciplina de Pneumologia da Escola Paulista de Medicina - Unifesp, São Paulo, SP.

1. Médico da Disciplina de Pneumologia da Universidade Federal de J uiz de Fora (UFJ F); Doutor em Medicina.

2. Coordenador da Residência Médica de Pneumologia do Hospital de Messejana, CE; Doutor em Medicina.

3. Médica da Unidade de Terapia Intensiva Respiratória do Hospital São Paulo - Unifesp; Mestre em Pneumologia.
4. Professor Adjunto e Chefe da Disciplina de Pneumologia da Escola Paulista de Medicina - Unifesp.

Endereço para correspondência - Bruno do Valle Pinheiro, Rua Benjamin Constant, 1.073/401 - 36015-400 - Juiz de Fora, MG. Tel. (32) 3213-6955; E-mail: hsbvp@ zaz.com.br

Recebido para publicação em 13/2/01. Aprovado, após revisão, em $9 / 10 / 01$. 
maximum airway and plateau pressures, and also to the static compliance. Oxygen consumption $\left(\mathrm{VO}_{2}\right)$ after neuromuscular blockade was $124 \pm 48$ in $\mathrm{VCV}$ versus $143 \pm 50 \mathrm{ml} / \mathrm{min}$ in PCV, $p=0.42$. In the assisted mode, there was a statistical trend of a higher $\mathrm{VO}_{2}$ in PCV (219 \pm 72 versus $154 \pm$ $67 \mathrm{ml} / \mathrm{min}$ in $V C V, p=0.06)$, that was associated with a statistical trend of a higher oxygen cost of breathing $(O C B)$ during assisted $P C V$, although without statistical significance $(31 \pm 77$ in VCV versus $75 \pm 96 \mathrm{ml} / \mathrm{min}$ in PCV, $p=0.23)$, and also in a lower PvO $(34 \pm 7$ in PCV versus $42 \pm 6 \mathrm{ml} /$ min in VCV, $p=0.02$ ). These occurred despite a higher maximum inspiratory flow in the assisted mode in PCV (58 \pm 9 versus $48 \pm 4 \mathrm{~L} / \mathrm{min}$ in VCV, $p=0.01)$. In both $V C V$ and PCV the institution of controlled ventilation reduced cardiac debit and $\mathrm{DO}_{2}$ in as much as $20 \%$ relative to the assisted

mode. Conclusions: The implementation of controlled ventilation improved the oxygen delivery/consumption relationship in this severe and with high $O C B$ acute lung injury model. The PCV offered no additional benefits to VCV and it was associated with a higher OCB during the assisted mode.

Descritores - Respiração artificial. Troca gasosa pulmonar. Volume de ventilação pulmonar.

Key words - Artificial respiration. Pulmonary gas exchange. Tidal volume.

\section{INTRODUÇÃO}

Persiste aberta a questão sobre se há vantagens no uso da ventilação pressão-controlada sobre a ventilação volume-controlada, sobretudo na síndrome do desconforto respiratório agudo (SDRA). A ventilação pressão-controlada e limitada é uma modalidade na qual o ventilador, disparado pelo paciente (ciclos assistidos) ou pelo tempo (ciclos controlados), emite um fluxo inspiratório de padrão desacelerado, determinando rápido aumento na pressão das vias aéreas. A pressão nas vias aéreas, previamente estabelecida, é mantida durante toda a inspiração, cuja duração também é predeterminada em função da ciclagem a tempo. Dessa forma, nos ciclos controlados, de modo diferente da ventilação ciclada a volume, o volume corrente e o fluxo inspiratório variam em função da pressão estabelecida, do tempo inspiratório e das características de resistência e complacência do sistema respiratório. Nos casos em que o ciclo for assistido haverá influência também do esforço do paciente(1).

Teoricamente, em função de um fluxo inicial alto para atingir rapidamente a pressão determinada, seguida de uma desaceleração do fluxo para manter a pressão constante nas vias aéreas durante toda a inspiração, a ventilação pressão-controlada melhoraria as trocas gasosas, otimizando a relação ventilação-perfusão. Além disso, com a ventilação pressão-controlada, ao contrário da volumecontrolada, tem-se a segurança de não haver hiperdistensão de alvéolos com melhor complacência, fato que poderia evitar maior lesão induzida pelo ventilador $(2,3)$. Entretanto, os resultados de estudos clínicos comparando as duas modalidades na SDRA são conflitantes, principalmente quando se utiliza a ventilação volume-controlada com padrão de fluxo desacelerado ${ }^{(4-8)}$.
Siglas e abreviaturas utilizadas neste trabalho

Cest - Complacência estática do sistema respiratório

COR - Custo de oxigênio da respiração

DC - Débito cardíaco

$\mathrm{DO}_{2}$ - Transporte de oxigênio

$\mathrm{f}$ - Freqüência respiratória

$\mathrm{FIO}_{2}$ - Fração inspirada de oxigênio

$\mathrm{PaO}_{2}$ - Pressão parcial de oxigênio no sangue arterial

$\mathrm{PaO}_{2} / \mathrm{FIO}_{2}$ - Relação entre a pressão parcial de oxigênio no sangue arterial e a fração inspirada de oxigênio

$\mathrm{PaCO}_{2}$ - Pressão parcial de gás carbônico no sangue arterial

PEEP - Pressão positiva ao final da expiração

Ppico - Pressão de pico em via aérea

Pplat - Pressão de platô em via aérea

$\mathrm{PVO}_{2}$ - Pressão parcial de oxigênio no sangue venoso misto

SDRA - Síndrome do desconforto respiratório agudo

$\mathrm{Ti}$ - Tempo inspiratório

VC - Volume corrente

VCV - Ventilação ciclada a volume

VCVA - Ventilação ciclada a volume assistida

VCVC - Ventilação ciclada a volume controlada

$\mathrm{VO}_{2}$ - Consumo de oxigênio

VPC - Ventilação com pressão constante

VPCA - Ventilação com pressão constante assistida

VPCC - Ventilação com pressão constante controlada

Além do que foi comentado acima, modalidades de suporte ventilatório parcial, com maior sincronia entre 0 paciente e o ventilador, têm sido desenvolvidas e aplicadas a fim de evitar atrofia muscular respiratória e até mesmo o uso excessivo de sedativos e bloqueadores neuromusculares. Por outro lado, o maior trabalho respiratório na insuficiência respiratória aguda acarreta aumento da demanda metabólica por oxigênio para a manutenção da respiração. Isso ocorre à custa de aumento do fluxo sanguíneo e/ou aumento da extração de oxigênio pelos músculos respiratórios ${ }^{(9)}$. Define-se como custo de oxigênio da respiração (COR) a quantidade de oxigênio consumida pelo organismo para manutenção da respiração. A ventilação mecânica tem efeitos importantes, tanto na oferta quanto no consumo de oxigênio global no organis- 
mo e, principalmente, sobre o coR. Este varia, normalmente, de 1 a $3 \%$ do consumo de oxigênio global do organismo, mas, na insuficiência respiratória aguda, pode chegar até a $23 \%{ }^{(10)}$. Assim, além de garantir as trocas gasosas, um dos principais objetivos da ventilação mecânica é o de diminuir o trabalho da respiração e redirecionar a oferta de oxigênio dos músculos respiratórios para outros órgãos e sistemas, como cérebro, fígado, coração. 0 uso da ventilação mecânica nos modos controlados ou nos assistidos ajustados adequadamente pode reduzir em até $61 \% \circ \operatorname{COR}^{(9,10)}$.

O utra possível vantagem da ventilação pressão-controlada em relação à volume-controlada seria a oferta de maior ou menor pico de fluxo inspiratório durante os ciclos assistidos, em função de maior ou menor esforço do paciente. Essa possibilidade de variação do fluxo inspiratório poderia diminuir o trabalho respiratório do paciente, reduzindo o consumo de oxigênio para executar a respiração, ou seja, diminuindo o $\mathrm{COR}^{(11,12)}$.

Como a ventilação volume-controlada e a ventilação pressão-controlada podem ser utilizadas nos modos controlado e assistido, utilizaremos, para fins de nomenclatura neste trabalho, os termos ventilação com pressão constante (VPC), que pode ser usada nos modos assistido (VPCA) ou controlado (VPCC), e ventilação ciclada a volume, igualmente nos modos assistido (VCVA) e controlado (VCVC).

Neste estudo objetivamos comparar, de forma prospectiva e randômica, em um modelo experimental de lesão pulmonar aguda em cães induzida por ácido oléico, as duas modalidades de ventilação em relação às trocas gasosas, mecânica respiratória, transporte e consumo de oxigênio e custo de oxigênio da respiração.

\section{Material e métodos}

\section{Preparação do experimento}

Os experimentos foram realizados no Laboratório de Pesquisa Experimental da Disciplina de Pneumologia da Escola Paulista de Medicina, Universidade Federal de São Paulo. Foram utilizados sete cães mestiços, machos, de peso entre 15 e $25 \mathrm{~kg}$, procedentes do Biotério Central da Escola Paulista de Medicina. Os experimentos foram realizados de acordo com os "Princípios Éticos na Experimentação" da União Internacional Protetora dos Animais e da lei 6.638, de maio de 1979. Após anestesia com tionembutal sódico ( $20 \mathrm{mg} / \mathrm{kg}$, por via endovenosa), os animais foram intubados por via orotraqueal com cânula Rusch no 8 e mantidos em ventilação mecânica (Bird $\left.8400 \mathrm{Sti}^{\circledR}\right)$. Doses adicionais de $5 \mathrm{mg} / \mathrm{kg}$ de tionembutal sódico, por via endovenosa, foram administradas segundo a necessidade. Os parâmetros iniciais da ventilação mecânica foram: modalidade assistido-controlada; cicla$\mathrm{da}$ a volume; $\mathrm{FIO}_{2}=1 ; \mathrm{VC}=12 \mathrm{ml} / \mathrm{kg} ; \operatorname{PEEP}=2 \mathrm{CmH}_{2} \mathrm{O}$; sensibilidade $=0,5 \mathrm{CmH}_{2} \mathrm{O}$; fluxo inspiratório necessário para estabelecer o tempo inspiratório de 0,6s. Como o objetivo deste estudo foi comparar duas modalidades ventilatórias em um modelo de lesão pulmonar aguda, não houve preocupação de estabelecer qualquer estratégia protetora da lesão pela ventilação. Sendo assim, foram utilizados volumes correntes altos e PEEPs baixos. O Ti de $0,6 \mathrm{~s}$ foi escolhido para evitar, em função da taquipnéia dos animais, a inversão da relação inspiração e expiração e/ou o desenvolvimento de auto-PEEP.

Através da veia jugular interna direita foi inserido um cateter arterial pulmonar para medidas de débito cardíaco e obtenção de sangue venoso misto. A artéria carótida direita foi cateterizada para a medida da pressão arterial sistêmica e obtenção de sangue arterial. A veia femoral direita foi cateterizada para a infusão de ácido oléico e hidratação do animal, através da infusão de soro fisiológico para manter a pressão capilar pulmonar entre $12 \mathrm{e}$ $15 \mathrm{mmHg}$.

\section{Protocolo experimental}

A lesão pulmonar aguda foi induzida através da infusão endovenosa de ácido oléico $(0,05 \mathrm{mg} / \mathrm{kg} \text {, em "bolus" })^{(13)}$. Após 60 minutos, gasometrias arteriais foram colhidas em intervalos de 30 minutos. Após duas gasometrias com $\mathrm{PaO}_{2} / \mathrm{FIO}_{2}$ inferior a $200 \mathrm{mmH} \mathrm{g}$, os animais foram randomizados para receber ou ventilação pressão-constante ou ventilação ciclada a volume. Em ambos os casos os animais eram mantidos por 40 minutos no modo assistido, seguidos de um curto período no modo controlado, após curarização com succinilcolina ( $1 \mathrm{mg} / \mathrm{kg}$, endovenosa). Sendo assim, duas seqüências de quatro estratégias ventilatórias poderiam ocorrer:

- $\mathrm{VPCA} \rightarrow \mathrm{VPCC} \rightarrow \mathrm{VCVA} \rightarrow \mathrm{VCVC}$

- $\mathrm{VCVA} \rightarrow \mathrm{VCVC} \rightarrow \mathrm{VPCA} \rightarrow \mathrm{VPCC}$

Durante todo o experimento a $\mathrm{FIO}_{2}$ foi de 1 ; a sensibilidade de $0,5 \mathrm{CmH}_{2} \mathrm{O}$ e a PEEP de $2 \mathrm{CmH}_{2} \mathrm{O}$. $\mathrm{Na}$ ventilação ciclada a volume, foi mantido o vc de $12 \mathrm{ml} / \mathrm{kg}$, com um fluxo suficiente para obter um tempo inspiratório de $0,6 \mathrm{~s}$, com padrão desacelerado. Durante o período de ventilação controlada, ajustava-se a freqüência respiratória igual à que 0 animal apresentava durante a ventilação assistida. Na ventilação com pressão constante, estabeleceramse os seguintes parâmetros: $\mathrm{Ti}=0,6 \mathrm{~s}$ e nível de pressão inspiratória ajustado para obter um vc de $12 \mathrm{ml} / \mathrm{kg}$, tanto no modo assistido quanto no controlado. Da mesma forma que na ventilação ciclada a volume, durante o período de ventilação controlada ajustava-se a freqüência respiratória igual à que 0 animal apresentava durante a ventilação assistida.

Após 40 minutos em cada modo de ventilação na forma assistida, foram realizadas gasometrias arteriais e venosas mistas, medidas de débito cardíaco (DC), transporte 
de oxigênio $\left(\mathrm{DO}_{2}\right)$ e consumo de oxigênio pelo princípio de Fick $\left(\mathrm{VO}_{2}\right)$. No modo assistido, todos os ciclos foram deflagrados pelo esforço do animal, sendo tal fato verificado pela inspeção visual do mesmo e pela análise da presença de deflexão negativa no início da inspiração no gráfico das curvas de pressão versus tempo. Após a paralisação e verificação de que todos os ciclos passaram a ser controlados, foram repetidas as gasometrias arteriais e venosas mistas e as medidas do transporte e do consumo de oxigênio. 0 custo de oxigênio para a respiração (COR) foi calculado pela diferença entre o consumo de oxigênio antes e após a curarização. Foram realizadas também medidas das pressões de pico (Ppico) e de platô (Pplat), utilizando-se uma pausa inspiratória de $2 \mathrm{~s}$, bem como medidas da complacência estática do sistema respiratório (Cest).

\section{Análise estatística}

As variáveis estudadas foram comparadas entre as modalidades através do teste $t$ de Student pareado e bicaudal, considerando-se diferenças estatisticamente significantes quando $p<0,05$.

\section{Resultados}

Foram estudados sete animais, sendo que em quatro deles a modalidade ventilatória inicial foi volume-controlada e em três, pressão-controlada. Os resultados das variáveis estudadas nas duas modalidades estão demonstrados na Tabela 1 (durante a ventilação assistida) e na Tabela 2 (durante a ventilação controlada).

$\mathrm{Não}$ houve diferenças em relação às trocas gasosas $\left(\mathrm{PaO}_{2}\right.$ e $\mathrm{PaCO}_{2}$ ) entre a ventilação ciclada a volume e a ventilação com pressão constante, tanto durante a ventilação assistida, quanto durante a controlada. Esses resultados estão ilustrados nos gráficos da Figura 1. Também não

\section{TABELA 1}

Variáveis estudadas na ventilação ciclada a volume (VCV) e na ventilação com pressão constante (VPC) no modo assistido (valores expressos em média desvio padrão)

\begin{tabular}{|c|c|c|c|}
\hline \multirow[t]{2}{*}{ Variáveis estudadas } & \multicolumn{2}{|c|}{ Modalidade de ventilação } & \multirow[t]{2}{*}{$\mathrm{p}$} \\
\hline & VCVA & VPCA & \\
\hline $\mathrm{PaO}_{2}(\mathrm{mmHg})$ & $76 \pm 28$ & $67 \pm 20$ & 0,17 \\
\hline $\mathrm{PaCO}_{2}(\mathrm{mmHg})$ & $45 \pm 12$ & $43 \pm 10$ & 0,48 \\
\hline $\mathrm{DC}(\mathrm{L} / \mathrm{min})$ & $3,02 \pm 0,78$ & $3,35 \pm 1,42$ & 0,28 \\
\hline $\mathrm{DO}_{2}(\mathrm{ml} / \mathrm{min})$ & $465 \pm 152$ & $490 \pm 254$ & 0,63 \\
\hline $\mathrm{VO}_{2}^{2}(\mathrm{ml} / \mathrm{min})$ & $154 \pm 67$ & $219 \pm 72$ & 0,06 \\
\hline $\mathrm{PvO}_{2}(\mathrm{mmHg})$ & $42 \pm 6$ & $34 \pm 7$ & 0,02 \\
\hline f (respirações por minuto) & $41 \pm 18$ & $40 \pm 10$ & 0,95 \\
\hline Pico de fluxo inspiratório (L/min) & $48 \pm 4$ & $58 \pm 9$ & 0,01 \\
\hline
\end{tabular}

houve diferenças no débito cardíaco e, conseqüentemente, no transporte de oxigênio, entre os dois grupos (Figura 2).

A complacência estática do sistema respiratório, bem como as pressões de pico e de platô, todas medidas após a paralisação dos animais, não foram diferentes entre os dois grupos (Tabela 2).

0 consumo de oxigênio medido após a curarização dos animais foi semelhante entre os dois grupos $(124+48$ na VCVC versus $143+50$ na VPCC, com $p=0,42)$. Entretanto, $0 \mathrm{VO}_{2}$ antes da paralisação foi maior durante a VPCA $(219+72$ na VPCA versus $154+67$ na VCVA, com $p=0,06)$. Embora isso tenha determinado um COR maior durante essa modalidade ventilatória em relação à VCVA, não houve diferença estatisticamente significante (31 + 77 na vCV versus $75+96$ na VPC, $p=0,23$ ) (Figura 3 ). Paralelamente, a $\mathrm{PvO}_{2}$ durante a VPC foi menor do que durante a vCV, na modalidade assistida ( $34+7$ versus 42 +6 , respectivamente, com $p=0,02$ ). Acompanhando 0 maior consumo de oxigênio e a menor $\mathrm{PvO}_{2}$ na VPCA em relação à VCVA, o pico de fluxo inspiratório durante os ciclos assistidos foi maior na ventilação com pressão constante $(58+9$ na VPCA versus $48+4$ na VCVA, com $p=$ $0,01)$.

A instituição da ventilação controlada através da curarização reduziu de forma significativa $0 \mathrm{DC}$ e $\mathrm{DO}_{2}$ em relação à ventilação assistida, tanto na ventilação ciclada a volume quanto na ventilação com pressão constante (Figura 4).

\section{DISCUSSÃo}

A ventilação com pressão constante ciclada a tempo (ventilação pressão-controlada) tem sido utilizada como opção de ventilação na SDRA, associada ou não à inversão da relação inspiração-expiração. Quando comparada

TABELA 2

Variáveis estudadas na ventilação ciclada a volume (VCV) e na ventilação com pressão constante (VPC) no modo controlado (valores expressos em média desvio padrão)

\begin{tabular}{lccc} 
Variáveis estudadas & \multicolumn{2}{c}{ Modalidade de ventilação } & $p$ \\
\cline { 2 - 3 } & VCVC & VPCC & \\
\hline $\mathrm{PaO}_{2}(\mathrm{mmHg})$ & $74 \pm 28$ & $67 \pm 20$ & 0,18 \\
$\mathrm{PaCO}(\mathrm{mmHg})$ & $44 \pm 12$ & $41 \pm 10$ & 0,13 \\
$\mathrm{DC}(\mathrm{L} / \mathrm{min})$ & $2,44 \pm 0,61$ & $2,60 \pm 0,70$ & 0,46 \\
$\mathrm{DO}_{2}(\mathrm{ml} / \mathrm{min})$ & $377 \pm 138$ & $375 \pm 126$ & 0,95 \\
$\mathrm{Cest}\left(\mathrm{ml} / \mathrm{cmH}_{2} \mathrm{O}\right)$ & $18 \pm 7$ & $19 \pm 7$ & 0,57 \\
$\left.\mathrm{Ppico}_{(\mathrm{cmH}} \mathrm{O}\right)$ & $19 \pm 4$ & $18 \pm 4$ & 0,13 \\
$\left.\mathrm{Pplat}_{(\mathrm{cmH}} \mathrm{O}\right)$ & $13 \pm 4$ & $14 \pm 3$ & 0,87 \\
$\mathrm{VO}_{2}\left(\mathrm{ml} / \mathrm{min}^{2}\right)$ & $124 \pm 48$ & $143 \pm 50$ & 0,42 \\
$\mathrm{PvO}_{2}(\mathrm{mmHg})$ & $41 \pm 12$ & $40 \pm 13$ & 0,72 \\
\hline
\end{tabular}




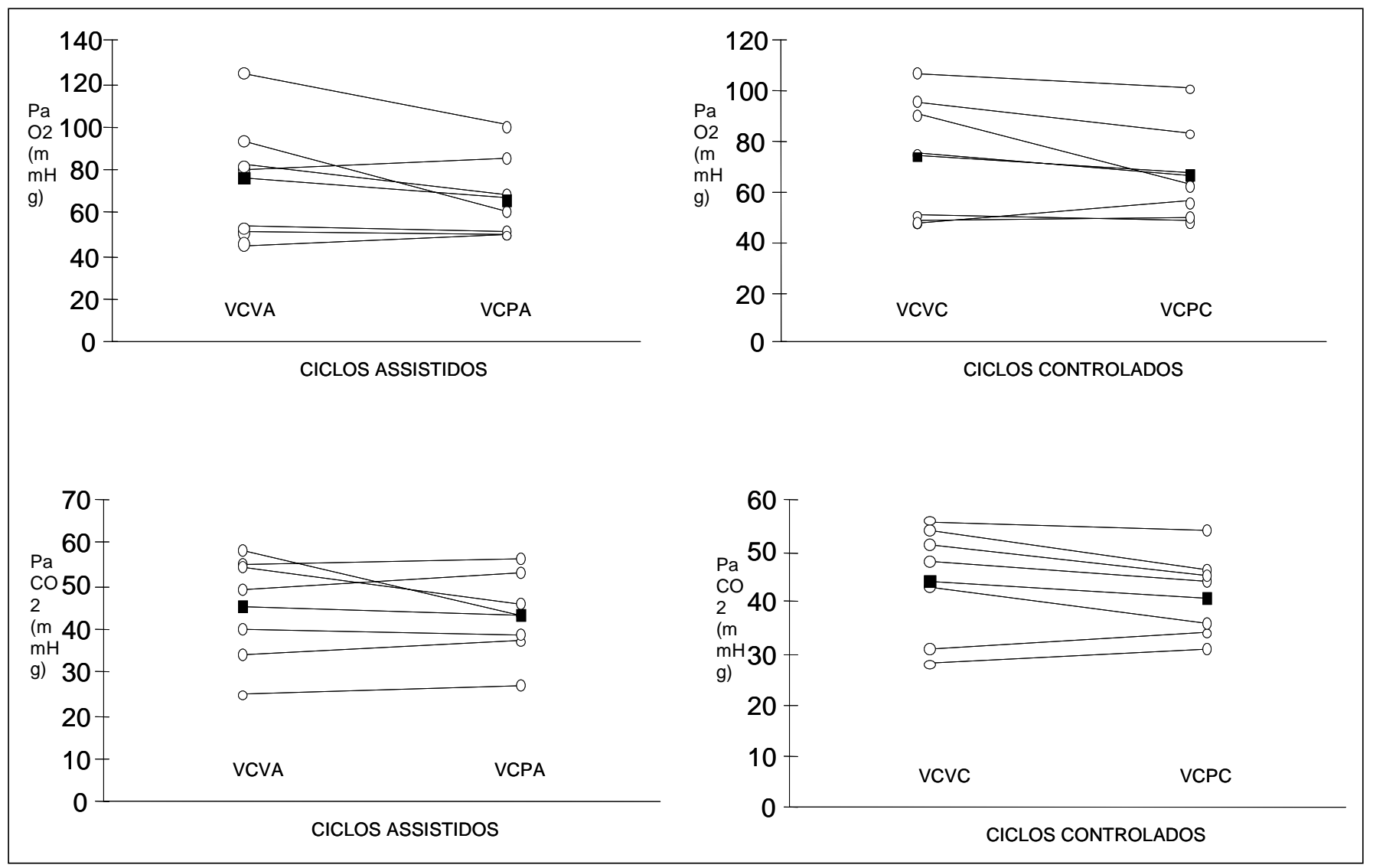

Figura 1 - Evolução da $\mathrm{PaO}_{2}$ e $\mathrm{PaCO}_{2}$ nos ciclos assistidos e controlados nas modalidades ventilatórias ciclada a volume (VCV) e com pressão constante (VPC). As linhas finas representam os valores de cada animal e as grossas, as médias de cada grupo. Não houve diferenças em relação à $\mathrm{PaO}_{2}$ e à $\mathrm{PaCO}_{2}$ entre as modalidades VCV e VPC, tanto nos ciclos assistidos, quanto nos controlados.

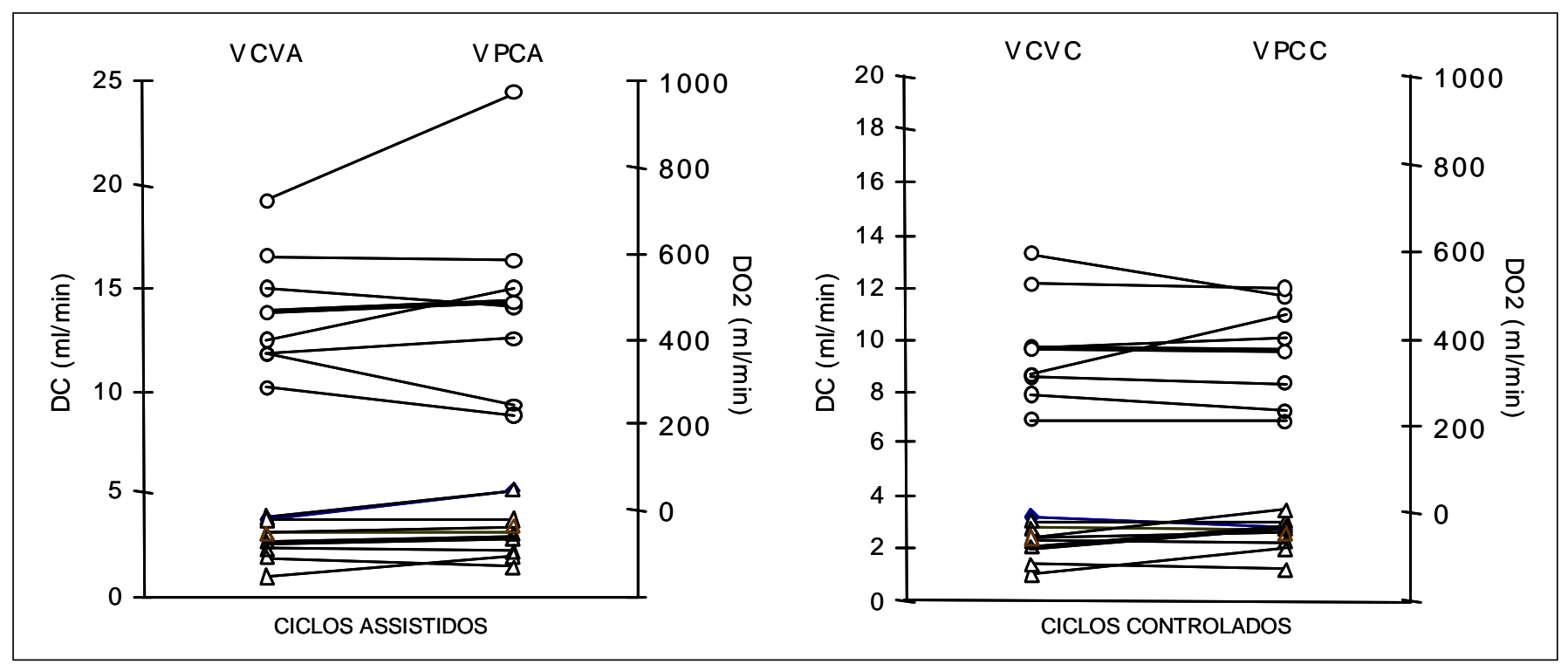

Figura 2 - Evolução do DC e do $\mathrm{DO}_{2}$ nos ciclos assistidos e controlados nas modalidades ventilatórias ciclada a volume (VCV) e com pressão constante (PC). As linhas finas representam os valores de cada animal e as grossas, as médias de cada grupo. Não houve diferenças em relação ao DC e ao $\mathrm{DO}_{2}$ entre as modalidades VCV e VPC, tanto nos ciclos assistidos, quanto nos controlados. 


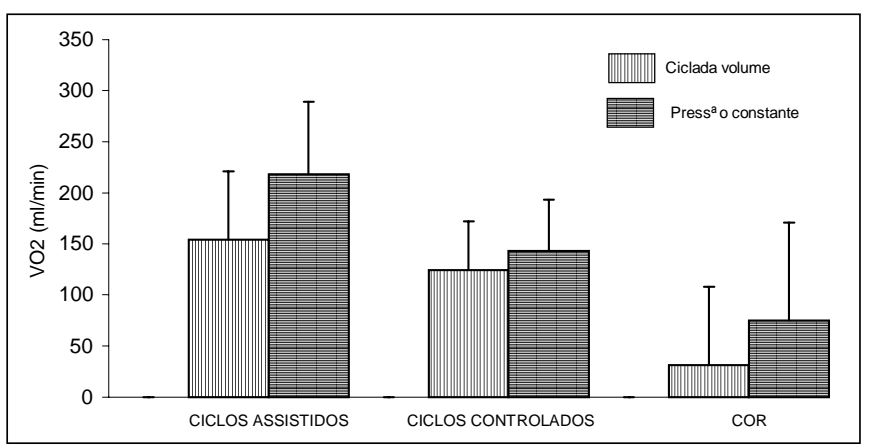

Figura 3 - Consumo de oxigênio nas modalidades ciclada a volume (VCV) e pressão constante (VPC) nos ciclos assistidos (154 67 versus $21972, p=0,06)$ e nos ciclos controlados (124 48 versus $14350, p=0,42$ ). O custo de oxigênio da respiração (COR) não foi significantemente diferente entre as duas modalidades (31 77 na VCV versus 7596 na VPC, $p=0,23)$.

à ventilação ciclada a volume (volume-controlada) com onda de fluxo quadrada, a VPC, em função de seu fluxo desacelerado, associa-se a melhora nas trocas gasosas, com melhor ventilação de alvéolos com maior constante de tempo. Entretanto, os trabalhos clínicos não demonstraram de forma definitiva as vantagens da VPC, sobretudo quando comparada com a vcV com fluxo de padrão desacelerado.

Neste modelo experimental, procuramos comparar a VPC e a vCV durante a ventilação de cães com lesão pulmonar aguda induzida por ácido oléico. 0 modelo experimental escolhido ocasionou uma lesão pulmonar aguda grave, com intenso comprometimento das trocas gasosas, com todos os animais evoluindo com relação $\mathrm{PaO}_{2} /$ $\mathrm{FIO}_{2}$ menor que $100 \mathrm{mmHg}$. Os ajustes nos parâmetros nas duas modalidades foram feitos para mantê-las similares, exceto pelo padrão de desaceleração do fluxo. $\mathrm{Na}$ VCV a desaceleração é predeterminada pelas características do ventilador, enquanto na VPC o fluxo inicial e o padrão de desaceleração dependem da diferença entre a pressão ajustada e a pressão alveolar, que, por sua vez, é influenciada pelo esforço inspiratório e pela impedância (resistência e complacência) do sistema respiratório.

A VPC não melhorou as trocas gasosas $\left(\mathrm{PaO}_{2}\right.$ e $\left.\mathrm{PaCO}_{2}\right)$ e os parâmetros de mecânica pulmonar em relação à vcV. Resultados conflitantes são encontrados na literatura quando são avaliados esses parâmetros nas duas modalidades de ventilação. Diferenças nos ajustes da ventilação e diferenças entre as populações estudadas podem contribuir para esse fato. Abraham e Yoshihara, estudando 10 pacientes com SDRA, demonstraram melhora na $\mathrm{PaO}_{2} \mathrm{Com}$ a VPC em relação à VCV com fluxo quadrado, mantendo os demais ajustes de ventilação constantes. Esses autores demonstraram menor pico de pressão inspiratória e maior complacência dinâmica durante a VPC, sem descrever,

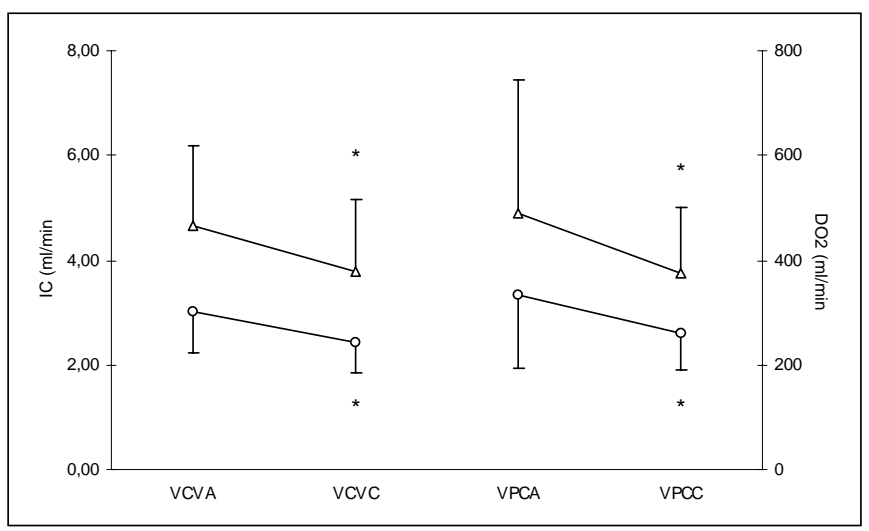

Figura 4 - Houve redução do índice cardíaco (círculos) após curarização na ventilação ciclada a volume (de 3,02 0,78 para 2,44 $0,61, p=0,01$ ) e na ventilação com pressão constante (de 3,35 1,42 para 2,60 0,70, $p=0,01$ ). O mesmo ocorreu com o transporte de oxigênio, com redução após curarização na ventilação ciclada a volume (de 465152 para $377 \quad 138, p=0,01$ ) e na ventilação com pressão constante (de 490254 para 375 126, $p$ $=0,03$ ).

entretanto, o comportamento da pressão de platô e da complacência estática nas duas modalidades(5). Em estudo semelhante, porém comparando VPC com VCV com fluxo desacelerado em 11 pacientes com insuficiência respiratória aguda (não necessariamente com SDRA), Muñoz et al. não encontraram diferenças tanto na $\mathrm{PaO}_{2}$, quanto na $\mathrm{PaCO}_{2}$. Além disso, eles mostraram que o pico de pressão medido através de um cateter no interior do tubo traqueal foi maior na VPC, sendo sempre alcançado ao final da inspiração. De forma diferente, o pico de pressão medido pelo ventilador foi maior na VCV, sendo muitas vezes alcançado no início da inspiração, quando um pequeno volume tinha sido ofertado ao paciente, sendo, portanto, em função da resistência oferecida pelo circuito inspiratório. Esses resultados questionam a importância clínica das diferenças entre as duas modalidades nas pressões de pico medidas pelo ventilador ${ }^{(6)}$. Estudando 12 pacientes com insuficiência respiratória aguda, não observamos diferenças na $\mathrm{PaO}_{2}$ e na $\mathrm{PaCO}_{2}$ durante a VPC, vCV com fluxo constante ou VCV com fluxo desacelerado. De forma semelhante a Muñoz et al., observamos menor pressão de pico nas vias aéreas com a VPC, conseqüentemente com melhor complacência dinâmica do sistema respiratório. A importância clínica desse achado, conforme já discutido, é questionável, em função de não termos medido a pressão no interior das vias aéreas ${ }^{(7)}$.

$M$ ais recentemente, Esteban et al. compararam, em estudo prospectivo, multicêntrico e randomizado, as duas modalidades ventilatórias em pacientes com SDRA. Não houve diferenças nos parâmetros gasométricos entre as duas modalidades. Os pacientes ventilados na modalida- 
de volume-controlada apresentaram maior mortalidade durante a internação na unidade de terapia intensiva, porém, no momento da randomização, havia maior freqüência de insuficiência renal e choque séptico entre esses pacientes, invalidando, de certa forma, esse resultado. Quando os autores realizaram uma análise multivariada para estabelecerem os fatores de risco para óbito entre todos os pacientes estudados, a ventilação na modalidade volume-controlada não figurou entre os fatores de risco de óbito(14). Sendo assim, até o momento, não há evidências sobre vantagens de uma modalidade sobre a outra, em relação à evolução final dos pacientes com SDRA.

O modelo experimental utilizado ocasionou lesão pulmonar aguda grave com intenso comprometimento da troca gasosa e da mecânica pulmonar, determinando elevado trabalho respiratório. Esse fato fica bem evidente quando se analisa o elevado custo de oxigênio da respiração tanto na VCVA $\left(30+77 \mathrm{ml} / \mathrm{min} / \mathrm{m}^{2}\right)$, quanto na VPCA $\left(76+96 \mathrm{ml} / \mathrm{min} / \mathrm{m}^{2}\right)$, bem superiores ao $\mathrm{VO}_{2}$ do diafragma de cães em condições basais, que é em torno de 0,2 a $0,8 \mathrm{ml} / 100 \mathrm{~g} / \mathrm{min}$. Em qualquer situação de insuficiência respiratória grave, seja por comprometimento da mecânica pulmonar, seja por aumento da demanda ventilatória, o consumo de oxigênio pelos músculos respiratórios aumenta muito. Em um modelo experimental de elevação do trabalho respiratório por aumento da resistência inspiratória em cães, Robertson et al. verificaram que 0 $\mathrm{VO}_{2}$ diafragmático pode chegar a até $24 \mathrm{ml} / 100 \mathrm{~g} / \mathrm{min}$. Deve-se considerar que a medida do con leva em conta atividade muscular não-respiratória, incluindo outros músculos esqueléticos, os quais podem contribuir para esses valores bastante elevados encontrados nos modelos experimentais(15). No nosso modelo, o maior consumo de oxigênio durante os ciclos assistidos, tanto na modalidade pressão constante, quanto na ciclada a volume, foi compensado por elevações paralelas no transporte de oxigênio e na taxa de extração de oxigênio. Esses mecanismos compensatórios são limitados e a partir desse limite, cujo nível dependerá da reserva cardiocirculatória do indivíduo, haverá prejuízo na perfusão de diferentes órgãos. Por essa razão, em pacientes críticos em insuficiência respiratória aguda grave, a presença de COR exagerado deve ser suspeitada e, caso ajustes adequados na ventilação assistida não sejam atingidos, a ventilação controlada deverá ser instituída.

Uma possível explicação para o maior $C O R$ e a menor $\mathrm{PvO}_{2}$ na VPCA em relação à VCVA no modelo aqui estudado pode estar relacionada ao modo como foi ajustado o nível de pressão na VPC. Este foi regulado para atingir o Vc desejado de $12 \mathrm{ml} / \mathrm{kg}$. No modo controlado com o animal curarizado, qualquer redução no nível de pressão programada acarretaria redução do Vc, desde que mantidas as variáveis de mecânica pulmonar. Entretanto, nos ciclos assistidos, a redução do nível de pressão ajustado acompanhar-se-ia de aumento compensatório do esforço muscular para manutenção do vc, gerando maior pico de fluxo inspiratório, mas à custa de maior trabalho respiratório e maior consumo de oxigênio. Portanto, achamos que, se a VPC for instituída para ventilação de pacientes em desconforto respiratório, o nível de pressão programado deverá levar em conta não só o VC, mas também o trabalho respiratório em eventuais ciclos assistidos deflagrados por esses pacientes. Na VPC, o trabalho respiratório exercido pelo paciente no modo assistido depende do ajuste dos níveis pressóricos, de forma semeIhante ao que ocorre na vcv, em que o trabalho exercido pelo paciente depende do ajuste do fluxo inspiratório.

$C$ abe ressaltar que a ventilação assistida, seja na forma de VCV ou VPC, pode ser muito pouco eficiente em reduzir o trabalho respiratório e o COR. Tal fato ressalta a importância da sedação e, se necessário, da curarização associadas à otimização dos ajustes do ventilador. No presente modelo o bloqueio neuromuscular teve significativo impacto sobre $0 \mathrm{DC}, \mathrm{DO}_{2}$ e $\mathrm{VO}_{2}$, com reduções da ordem de $20 \%$ nestas variáveis, equilibrando a relação oferta/ consumo de $\mathrm{O}_{2}$ e, possivelmente, redirecionando a oferta de $\mathrm{O}_{2}$ a outros órgãos, como rins, fígado e cérebro ${ }^{(16)}$.

Concluímos que, em um modelo de insuficiência respiratória grave, com grande consumo de oxigênio pela respiração, a manutenção da ventilação mecânica controlada em comparação com a assistida melhorou a relação entre a oferta e o consumo de oxigênio. Observamos que a ventilação com pressão constante, em relação à ciclada a volume, não trouxe benefícios nas trocas gasosas ou na mecânica pulmonar, podendo, em condições de ajustes inadequados dos seus parâmetros, aumentar o consumo de oxigênio pela respiração.

\section{RefERÊNCIAS}

1. Branson RD, Chatburn RL. Technical description and classification of modes of ventilator operation. Respir Care 1992;37:1026-44.

2. Blanch $P B$, Jones $M$, Layon AJ, et al. Pressure-preset ventilation. Part 1: Physiologic and mechanical considerations. Chest 1993;104:5909.

3. Blanch PB, Jones M, Layon AJ, Camner N. Pressure-preset ventilation. Part 2: Mechanics and safety. Chest 1993;104:904-12.

4. Al-Saady N, Bennett ED. Decelerating inspiratory flow waveform improves lung mechanics and gas exchange in patients on intermittent positive-pressure ventilation. Intensive Care Med 1985;11:68-75.

5. Abraham E, Yoshihara G. Cardiorespiratory effects of pressure controlled ventilation in severe respiratory failure. Chest 1990;98:14459 .

6. Muñoz J, Guerrero JE, Escalante J L, Palomino R, De La Calle B. Pressure-controlled ventilation versus controlled mechanical ventilation with decelerating inspiratory flow. Crit Care Med 1993;21:1143-8.

7. Holanda MA, Pinheiro BV, Larges $C M$, et al. Volume assured pressure support (VAPS) ventilation in comparison with volume assisted with constant flow (VACF), volume assisted with decelerating flow wave- 
form (VADF) and with pressure assisted-controlled (PAC) ventilation in patients with respiratory failure [Abstract]. Am J Respir Crit Care Med 1997;155:A527.

8. Amorim FF, Carrilho J, Holanda MA, et al. Pressure-controlled ventilation (PCV) versus volume-controlled ventilation (VCV) with decelerating flow waveform in patients with respiratory failure with or without airway obstruction [Abstract]. Am J Respir Crit Care Med 1997;155: A530.

9. Russell JA. The effects of mechanical ventilation on oxygen delivery and oxygen consumption. In: Marini JJ, Slutsky, AS. Physiological basis of ventilatory support. New York: Marcel Dekker, 1998;345-74.

10. Manthous CA, Hall J B, Kushner R, Schmidt GA, Russo G, Wood LDH The effect of mechanical ventilation on oxygen consumption in critically ill patients. Am J Respir Crit Care Med 1995;151:210-4.

11. Cinnella G, Conti G, Lofaso F, Lorino H, Harf A, Lemaire F, et al. Effects of assisted ventilation on the work of breathing: volume-controlled versus pressure-controlled ventilation. Am J Respir Crit Care Med 1996;153:1025-33.
12. Kanak R, Fahey PJ, Vanderwarf C. Oxygen cost of breathing. Changes dependent upon mode of mechanical ventilation. Chest 1985;87: 126-7.

13. Mutch WA, Harms S, Lefevre GR, Graham MR, Girling LG, Kowalski $\mathrm{SE}$. Biologically variable ventilation increases arterial oxygenation over that seen with positive end-expiratory pressure alone in a porcine model of acute respiratory distress syndrome. Crit Care Med 2000;28:245764.

14. Esteban A, Alia I, Gordo F, de Pablo R, Suarez J, Gonzalez G, et al. Prospective randomized trial comparing pressure-controlled ventilation and volume-controlled ventilation in ARDS. Chest 2000;117:1690-6.

15. Robertson J r CH, Foster GH, J ohnson J $r$ RL. The relationship of respiratory failure to the oxygen consumption, of lactic production by and distribution of blood flow among respiratory muscles during increasing respiratory resistance. J Clin Invest 1977;59:13-42.

16. Viires N, Sillve G, Aubier M, Rassidakis A, Roussos C. Regional blood flow distribution in dog during induced hypotension and low cardiac output. Spontaneous breathing versus artificial ventilation. J Clin Invest $1982 ; 72: 935-47$. 